\title{
Les inuit ou l'impossible métissage!
}

\section{Etienne Bours}

\section{(2) OpenEdition \\ Journals}

\section{Édition électronique}

URL : http://journals.openedition.org/ethnomusicologie/685

ISSN : 2235-7688

Éditeur

ADEM - Ateliers d'ethnomusicologie

Édition imprimée

Date de publication : 1 janvier 2001

Pagination : 77-90

ISBN : 2-8257-0723-6

ISSN : 1662-372X

Référence électronique

Etienne Bours, «Les inuit ou l'impossible métissage! », Cahiers d'ethnomusicologie [En ligne], 13 | 2001, mis en ligne le 09 janvier 2012, consulté le 02 mai 2019. URL : http://journals.openedition.org/ ethnomusicologie/685

Ce document a été généré automatiquement le 2 mai 2019.

Tous droits réservés 


\title{
Les inuit ou l'impossible métissage!
}

\author{
Étienne Bours
}

1 Les Inuit, peuple idéal ou peuple idéalisé? Les Inuit que l'on voudrait derniers gardiens du tambour sacré, que l'on espère farouches défenseurs d'un chant ancestral ancré dans la toundra et éternellement scandé de ses ayaya nostalgiques. Là-bas, tout au Nord de nos rêves, vivent plus de 100000 Inuit. Ils sont devenus champions des métissages en tous genres, cultivant adroitement le sens de l'adaptation aux changements. Les «derniers rois de Thulé» ${ }^{1}$ sont depuis longtemps les premiers rois de l'adoption de nouvelles conditions. A l'écoute, les sens en éveil, le regard volontiers narquois, l'Inuk a toujours observé et a su prendre, sélectionner, ce qui lui paraissait bon pour améliorer son existence. Celle-ci est devenue, au fil des décennies, un amalgame étonnant de modernité et de traditions millénaires. Sans pour autant, d'ailleurs, présenter exactement les mêmes développements dans chaque partie de l'Arctique. Les Inuit du Groenland, ceux des territoires américains et ceux de Sibérie ne vivent évidemment pas tous les mêmes conditions à la même vitesse. Canada, Alaska et Groenland sont cependant devenus des endroits riches où l'enfer du Nord et le confort du Sud se sont téléscopés depuis longtemps déjà. La vie s'est faite métissage absolu. Maison chauffée au maximum contre igloo en période de chasse ou tente dès le printemps. Moto-neige ou chien de traîneau, pizza surgelée ou caribou cru, abrutissement devant la télévision ou connaissance extrême de la nature, individualisme importé contre sens de la communauté, chômage et assistance remplaçant la lutte pour la survie. La vie des Inuit a basculé avec les arrivées successives des Blancs: les marins, les baleiniers, les explorateurs, les missionnaires, les représentants des gouvernements, les enseignants. Chacun leur a apporté son lot de nouveautés, son sac de changements. Tracasseries administratives, apprentissage de langues nouvelles, sédentarisation, scolarisation, conversions à de nouvelles croyances... Ce qui avait débuté en douceur avec un peu de thé, du tabac et quelques fusils finit par s'imposer partout massivement et catégoriquement. Et les Inuit de s'en servir avec pragmatisme et intelligence, sans nécessairement perdre l'essentiel. 


\section{Musiques isolées}

2 On pourrait imaginer, dès lors, qu'en matière d'expressions musicales, le même sens de l'adaptation a opéré en faveur de métissages semblables à ceux de la vie de tous les jours. Soit une rencontre entre le chant ancestral, le pisiq ou chant personnel, et la chanson occidentale: une modernisation de l'un au contact de l'autre, comme un moule nouveau pour une pensée et une expression fondamentalement inchangées. Ce serait trop simple. Leurs traditions anciennes ont, pour toute une série de raisons, eu à souffrir en profondeur des apports nouveaux. Les plus insidieux de ceux-ci furent certainement les apports religieux. Le bagage des missionnaires regorgeait de sentences et de principes aux conséquences irrémédiables. En jouant à la fois sur les craintes, les promesses et les espoirs, catholiques et protestants luttèrent avec acharnement contre un ensemble complexe de croyances et de pratiques, contre le rôle central du chamane, contre le pouvoir du tambour, contre le caractère sacré du chant. Tout comme ils luttèrent contre la danse à tambour et le déchaînement collectif qu'elle pouvait provoquer. Ils remplaçaient un système d'équilibre social et animiste par un autre système, somme toute plus confortable et assorti de mille promesses de rédemption et autre bonheur éternel. Le chant Inuit et le tambour devaient en souffrir. Même s'il ne faudrait guère généraliser le propos et notamment souligner le fait que certains missionnaires furent, par contre, des artisans essentiels d'un renouveau des traditions ${ }^{2}$, il n'en reste pas moins qu'en nombre d'endroits celles-ci perdirent leur vigueur ou leur sens. Les musiques inuit furent sans doute isolées, condamnées à n'être pratiquées que sous contrôle ou à l'abri des regards des nouveaux arrivants. Celles qui étaient encore pratiquées entre eux conservaient donc toute leur énergie, leur originalité (elles étaient déjà isolées avant l'arrivée des Blancs), et n'avaient évidemment aucune raison de se métisser.

3 Pourtant, discrètement, les musiques occidentales faisaient une percée. Les chants et danses des marins ou des commerçants apportaient une autre gamme, un autre type de mélodie qui allait se glisser au sein de quelques expressions inuit et s'installer dans l'horizon musical des chanteurs eskimos même les plus «traditionnels». On abordera quelques exemples dans le détail.

4 Moins discrèt, par contre, était le débarquement des musiques occidentales au grand jour, celles qui s'installaient dans les pratiques arctiques, telles qu'elles étaient arrivées ou tout simplement légèrement adaptées par ces nouveaux pratiquants. On vit venir, selon les endroits, des instruments qui ne devaient pas tarder à fasciner les Inuit naturellement curieux et amateurs de musiques. Le violon, dont ils firent d'abord des copies grossières avec les moyens que leur offrait éventuellement la nature, l'harmonica, la guimbarde métallique, les cuivres, l'accordéon diatonique, la guitare, etc. Ici et là, certains de ces instruments s'imposèrent, bénévolement ai-je envie de dire c'est à dire sans prosélitisme, avec un répertoire pré-existant. C'est le cas de l'accordéon qui, tant au Canada qu'au Groenland, allait servir une musique de danses d'origines européennes. Polka (kalatuut) au Groenland, quadrilles au Canada, l'Inuk y a gagné une autre musique, une passion pour un instrument, pour d'autres danses. C'est une tradition nouvelle, devenue tradition inuit, surtout au Groenland où elle se transmet déjà de génération à génération. "The musicians are part of a tradition, where they have been taught melodies and songs from their parents, who often learned it from their parents», dit la notice du récent CD « Traditional Northgreenlandic Accordion Music» ${ }^{3}$. Historiquement, les Inuit ont appris ces airs 
et cet instrument via leurs contacts avec les marchands et marins, Ecossais dans la Baie d'Hudson, Danois et autres Européens au Groenland. Les autres instruments furent appris de la même manière mais firent également souvent partie de la panoplie de l'éducation trimballée par enseignants et missionnaires. Jennes faisait par exemple remarquer pour le Labrador que le village band (fanfare inuit) jouait également pendant les offices religieux ${ }^{4}$. Labrador où même certains prêtres ont appris Bach à leurs ouailles. Plus l'installation du Sud se développe au Nord et plus on verra de nouveaux instruments aux mains des Inuit et avec eux de nouvelles musiques, c'est à dire les musiques que ces instruments ont véhiculées dans leur sillage. Et c'est avec un réel bonheur que l'Eskimo s'adonne à cette pratique novatrice. Il suffit de se rappeler le film Nanook de Flaherty et l'émerveillement devant le phonographe ou encore le film que Claude Massot réalisa sur Flaherty et le tournage de Nanook (Qaloonak); on y voit Flaherty initier une Inuk au piano. Mais il faut se rappeler aussi du fait que, corrollaire effrayant de cette arrivée massive de nouveaux instruments, le tambour fut interdit en maints endroits, au point de parfois disparaître définitivement.

5 Au delà des instruments, les musiques de l'autre monde s'imposèrent par le chant. Les hymnes bien sûr et le chant choral qui s'est également fortement implanté au Groenland. Chants religieux, expression de la ferveur, ils ont été facilement asborbé par ce peuple pour qui spiritualité et vie n'ont jamais fait qu'un. Syncrétisme ou foi nouvelle? Un amalgame des deux sans doute, un réel métissage religieux mais une musique nouvelle une fois de plus. Ce sont en effet les hymnes, leurs mélodies et leur organisation chorale qui font aujourd'hui partie des nouvelles musiques inuit, même si de temps à autre, on décèle encore une façon de chanter, un abandon total dans la ferveur exprimée par ce chant, qui est bien plus inuit qu'occidentale 5 . La norme, par contre, est des plus rigide, copie conforme de n'importe quelle chorale religieuse.

Quant aux autres chansons occidentales, elles sont arrivées petit à petit, avec les enseignants, avec la radio, la télévision et enfin le disque. On entend alors à travers l'Arctique quelques exemples navrant $d u$ bon progrès distillé aux petites têtes encapuchonnées, comme «Frère Jacques» chanté en inuktitut! Le chanteur de l'Arctique saura se servir de ces chansons, il leur applique souvent un procédé traditionnel ancien qui consiste à reprendre une mélodie déjà connue et lui confier de nouvelles paroles. Tradition ancienne et nouvelle musique font alors bon ménage mais l'expression choisie n'en est pas moins empruntée aux étrangers puisque ces chansons s'accompagnent à la guitare et sont coulées dans un moule folk ou rock. Le chanteur Tumasi Quissa d'Akulivik, par exemple, décrit les plaisirs de la chasse sur l'air des «Chevaliers de la table ronde» 6 !

7 Musiques isolées, les expressions traditionnelles inuit le sont restées à travers ces changements et ces absorptions de nouvelles musiques. Les chants à tambour, les danses collectives, les chants de jeux, les expressions chamaniques ont souvent continué d'exister, plus isolées que jamais et surtout de plus en plus menacées. Mises en danger, parfois en voie de disparition, elles ont lutté à armes inégales contre le lot effarant de nouvelles expressions qui débarquaient comme autant de nouveaux jeux, accompagnées d'instruments, de techniques et de rêves. Elles n'ont jamais complètement disparu, même si elles ont souvent perdu leur place et leurs fonctions essentielles dans la société; mais elles n'ont jamais non plus tenté des expériences de métissage telles que celles qu'on rencontre en beaucoup d'autres régions du monde. 


\section{L'impossible métissage}

Comme je l'écrivais déjà en $1991^{7}$, «les genres musicaux se mélangent à peine. D'un côté, les traditions sont toujours pratiquées, comme on l'a vu, avec des changements qui affectent essentiellement leurs fonctions, le statut du musicien, etc. D'un autre côté, les nouvelles musiques voient le jour avec une grande rapidité, mais il y a très peu de mélanges entre ces deux types d'expression. Le compartimentage est la règle». Olsen (1972) le disait pour le Groenland: «la réaction normale à la musique occidentale a été de l'accepter ou de la rejeter, mais pas de la combiner avec la musique traditionnelle. Ce mélange musical sera rarement rencontré, mais il existe, particulièrement dans les régions à l'extrême nord et au sud du Groenland occidental». Johnston (1976) explique le même phénomène pour l'Alaska: «le chercheur peut entendre le même chanteur chanter des chants traditionnels et des chants harmonisés, mais nulle part vous n'entendrez des mélodies et rythmes eskimos altérés». Les exemples pour comprendre ce compartimentage étaient déjà nombreux il y a dix ans. Il était par exemple étonnant de voir le succès délirant de quelques chants à tambour joués en plein milieu d'un festival plutôt rock et chanson à l'Est du Groenland. Public et artiste étaient visiblement en symbiose totale pendant ce bref moment d'expression inuit ancestrale ${ }^{8}$. Rien n'a changé depuis dix ans sinon que les productions de musiques «modernes»se multiplient: accordéonistes, chanteurs pop, groupes rock et autres copies de l'Occident. On joue la musique traditionnelle ou on joue une des nouvelles musiques. Mais on ne joue pas la tradition revisitée au contact des nouveaux apports. La fusion est rarissime. Quelques chanteurs ont simplement décidé d'accompagner l'un ou l'autre ancien chant déjà mélodique avec une guitare et une section rythmique. D'autres ont risqué un ayaya en refrain typiquement eskimo dans une composition folk ou rock. On ne chante pas un chant traditionnel avec un accordéon et encore moins un chant actuel, nouvelle composition, avec le tambour traditionnel.

Cette règle est-elle absolue? Et si oui, pourquoi?

Sans entrer dans une analyse profonde de ce qu'est un métissage, on peut toujours déceler de discrets mélanges, quelques colorations nouvelles, parfois sournoises, parfois habiles, qui sont aux musiques inuit ce que de flagrants changements sont parfois à d'autres musiques. Les changements en question sont des métissages en ce sens qu'ils viennent de l'extérieur, ils sont le résultat de la rencontre entre deux cultures différentes. Ces timides métissages ne sont jamais érigés en système ou en école, ils apparaissent ici et là au gré des artistes, au fil du temps. Ils ressemblent à des accidents de l'histoire, ce que devrait être le métissage le plus intéressant - par opposition aux métissages provoqués par des conditions de marché de la musique. On peut au moins en aborder quelques exemples dans les chants de gorge et dans les chants personnels.

\section{Chants et jeux de gorge}

Depuis longtemps, sans doute, les chants de gorge et particulièrement les fameux kattajjait du Canada agissent comme des éponges. Leurs fonctions multiples, à la foi ludiques et éducatives, en font une sorte de réceptacle dans les limites duquel sont autorisés quelques emprunts, modifications, inspirations. La société évoluant, le katajjaq suit. Les femmes de Povungnituq chantent, par exemple, un chant de gorge qui décrit un

Cahiers d'ethnomusicologie, 13 | 2011 
ivrogne (Imialuttoq) ${ }^{9}$; il ne peut s'agir que d'une inspiration relativement récente. Il existe un certain nombre de kattajjait dits «mélodiques». Non contentes d'émettre des sons de gorge, des imitations de cris et de bruits, les femmes y développent également une courte mélodie qu'elles se répartissent en hoquet, faisant se succéder rapidement les accents forts qu'elle alternent en déphasage l'une par rapport à l'autre avec les accents faibles. Cette succession rapide construit une mélodie partagée. Comme le dit Jean Jacques Nattiez dans la notice de livret du CD «Chants et jeux des Inuit» ${ }^{10}$, «il semble que la plupart des kattajjait n'aient pas de mélodie propre, au sens européen du terme, mais ceux que, dans cette acception, nous avons appelés «kattajjait mélodiques «paraissent les emprunter à des chants connus». Ces mélodies viennent alors de chants utilisés pour les enfants ou d'hymnes appris des missionnaires. Tels sont dès lors les petits changements induits par les métissages de sociétés. Les chants de gorge n'ont guère évolué vers d'autres changements. Ils ont peut-être failli disparaître et ont revu le jour grâce notamment à un pasteur Inuit, ils ont ensuite bénéficié de l'effet de surprise qu'ils provoquent sur tout étranger et ont joui d'un important mouvement de «revival» au sein des communautés Inuit du Nouveau Québec et de la Terre de Baffin. Les jeunes apprennent des anciennes, le jeu a changé de fonction mais il reste jeu au sein des familles et des fêtes locales. Il amuse, il fait rire, il détend. Il est symbole d'une culture très locale, très précise et très appréciée des Inuit eux-mêmes. En d'autre termes, il va bien et n'a guère besoin d'apports extérieurs. C'est pourtant une notion récente et très occidentale, celle de spectacle, qui contribue à lui donner ce nouveau souffle d'aujourd'hui. Les chanteuses tournent en effet à travers le monde, jouant sur de grandes scènes devant des publics enthousiastes venus assister à un spectacle «musical». Compte tenu de ce réel métissage dans la fonction et de cet incroyable succès, on aurait pu penser que le katajjaq allait s'adonner à quelques expériences hybrides retentissantes ou à quelques métissages osés mais peut-être réussis. Il n'en est quasi rien. Sinon le disque du duo Tudjaat ${ }^{11}$, deux jeunes chanteuses de kattajait qui ont opéré une sorte de collage entre schémas de chants de gorge et chansons de type occidental. Soit cinq chansons pop entrecoupées de quelques effets extraits de kattajait connus, le disque se terminant par une succession de kattajjait traditionnels. Une entreprise pas très novatrice, même si pionnière dans le genre, un essai typique de ce que la vague world music pousse à faire aujourd'hui; mais un manque évident d'originalité et de réelle inventivité. Le tout ressemble à une superposition de deux genres mais pas à une réelle intégration de l'un dans l'autre. En dehors de ces maigres expériences, les différents chants de gorge se maintiennent tels quels. Mais relevant du domaine du jeu, il ne serait pas étonnant qu'ils soient encore soumis aux traitements les plus extravagants. 
Fig, 1: Kattajjaq par Mary Igaluk et Nellie Echalook. Inukjuak.

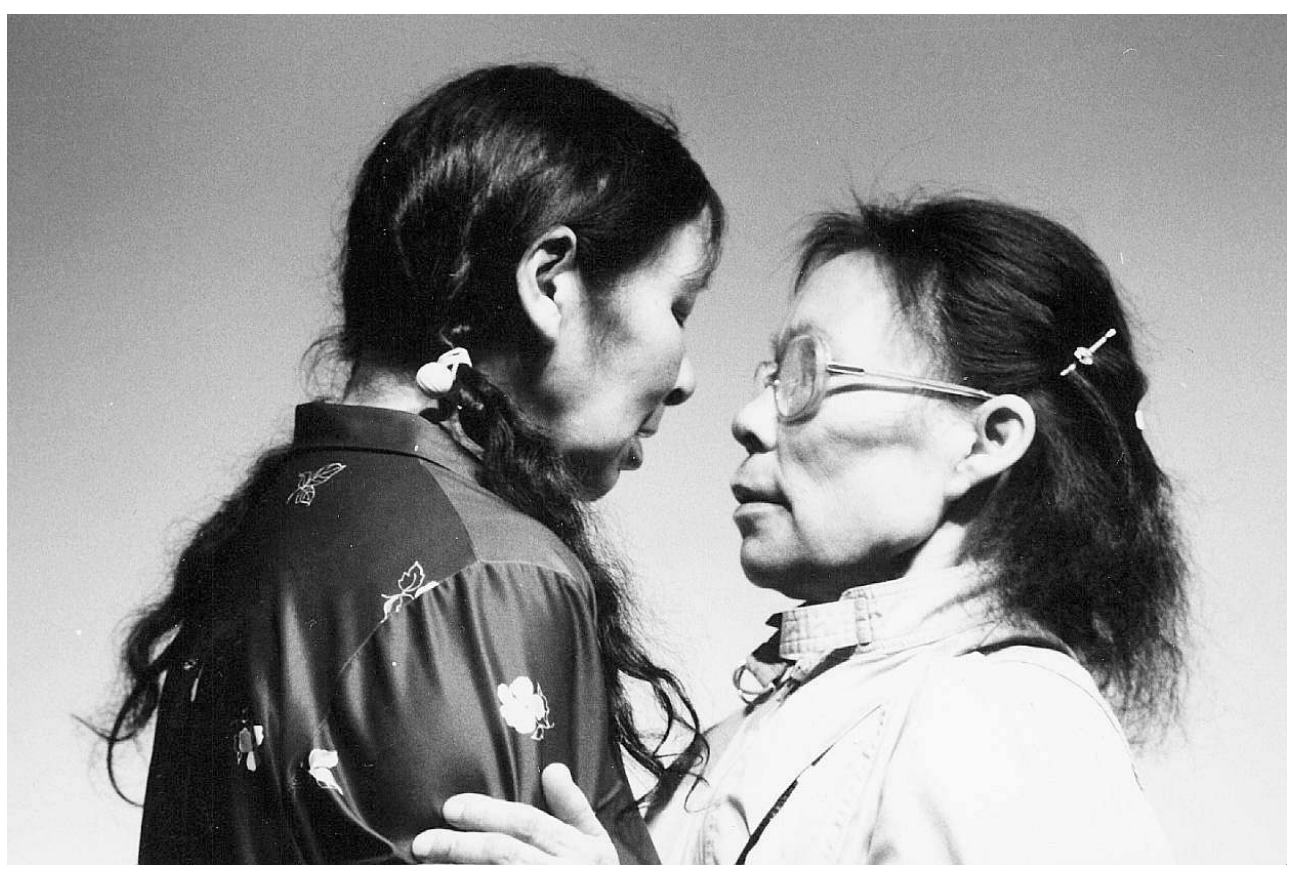

Photo: Étienne Bours.

\section{Le pisiq, chant personnel}

Le chant à tambour ou chant personnel est un phénomène plus complexe, plus important, avec lequel, manifestement, on ne joue pas. Le chant est celui de l'homme, il raconte ses expériences personnelles, ses impressions, ses chasses et ses pêches mais aussi ses craintes ou ses doutes. Il se chante pour la communauté, avec ou sans tambour, avec l'aide de la femme du compositeur qui en retient les paroles. Il est l'histoire et le journal $\mathrm{du}$ peuple. Il est un langage et utilise d'ailleurs, traditionnellement, une syntaxe différente de celle du langage parlé. «Le but de la musique est d'apprendre et non de répondre à une notion de divertissement», disait Donald Suluk ${ }^{12}$. Un chant ne peut se répéter sans être compris, il est né dans des conditions particulières et ne doit peut-être plus être chanté en dehors de celles-ci. Suluk s'insurgeait contre l'idée d'interpréter un chant ancien à la guitare. Chant personnel chez les uns, chant de cohésion du goupe chez les autres (groupes de chasseurs en Alaska et Sibérie), le chant à tambour est souvent une expression ritualisée. Il peut être associé à des cérémonies à caractère sacré dont la danse fait également partie. Il peut être un symbole d'unité, un rassemblement autour du tambour et de la parole d'un chasseur, il est un vecteur de liens privilégiés au travers de systèmes complexes pouvant lier deux chasseurs entre eux (igluriik ou iglua). Entre ces partenaires se déroulaient des joutes de chants, chants de moquerie, balance sociale, exutoire indispensable comme l'était cette relation privilégiée entre hommes pourvoyeurs de nourriture qui pouvaient alors vivre un échange de femmes et briser la consanguinité. Le chant a servi à règler des conflits, à remettre les fauteurs de trouble à leurs places, à guérir les malades, à rencontrer les esprits... On comprend dès lors qu'une série de tabous protègent tant bien que mal l'expression type des Inuit autant que leur tambour. Ce qui n'a pas empêché le pisiq d'évoluer ou de s'autoriser quelques emprunts 
ou quelques détours, légers bien sûr. Il ne se chante plus dans les mêmes conditions, il a perdu ses fonctions ancestrales mais il semble que certains chanteurs continuent d'en composer. Jean-Jacques Nattiez en donne un exemple des plus intéressants dans le CD Inuit Iglulik ${ }^{13}$. Le chanteur, Awa de Pond Inlet, raconte une expérience personnelle. Il chante le plaisir d'avoir été camper au printemps, d'avoir découvert de nombreux animaux autour de sa tente. Il termine son chant en chantant «Je suis si content de partir camper et de faire tout ce qui me plaît. Je suis si content de voyager et de passer la nuit à différents endroits. Ceci est le chant que j'ai composé moi-même. Je m'excuse de ne pas pouvoir jouer de la guitare bien que je puisse jouer de l'harmonica». Outre le fait que, comme le fait remarquer Nattiez, la mélodie est très clairement inspirée de la musique tonale occidentale, il est particulièrement intéressant de relever la dernière phrase de ce chant exécuté a cappella. Le chanteur regrette de ne pas jouer de la guitare. En d'autres termes, s'il savait en jouer, peut-être ce chant serait-il devenu une chanson folk, un pisiq à l'occidentale, racontant des expériences personnelles dans un moule occidental. C'est important à comprendre parce que Awa est ici doublement influencé par les musiques européennes et américaines: la mélodie d'une part et le besoin d'instruments nouveaux d'autre part. Métissage imperceptible que celui-ci mais évolution évidente d'un style traditionnel au contact des autres musiques. Exemple rarissime par contre, parce qu'il est, comme le laisse entendre cette dernière phrase, à la frontière des genres. Ajoutez à la mélodie effectivement très occidentale la guitare que semble regretter Awa et le chant bascule du côté de la chanson conforme à ce qui se fait à travers le monde. Peut-être pas en termes de composition et d'inspiration mais certainement en termes musicaux. Où est alors le chant à tambour qui chante un texte d'aujourd'hui, une préoccupation actuelle? Il est bien plus facile de trouver la formule inversée: un texte grave, actuel, profond, une réflexion, une expérience relatée en chanson avec guitares, basse, batterie et claviers. Soit le sens du verbe et de son importance en chanson, l'idée que chanter doit servir à distiller ses expériences, ses doutes, ses espoirs, comme idée rescapée intacte de la tradition. Mais le tambour relégué, oublié ou timidement écarté, comme s'il était tabou ou comme si la guitare s'avérait indispensable pour se faire entendre. Le tambour, pourtant, totalement vénéré, respecté, glorifié; un tambour que l'on n'hésite pas à chanter, sans en jouer! «Sois conscient du pouvoir de la nature, elle est la vraie source de vie. Tu dois toimême raviver le son mourant du tambour», chante le groupe groenlandais Silamiut dans une chanson intitulée «Inngerpalaaq» ${ }^{14}$. Mais cette chanson est une chanson rock dont le son du tambour traditionnel est complètement absent. Et si d'aventure certains jeunes musiciens décident d'incorporer le tambour dans leurs compositions nouvelles, c'est de sampling, de collage, de superposition qu'il s'agit. Le groupe Sume du Groenland le fit à ses débuts, commençant une pièce par un enregistrement de tambour traditionnel joué par Egon Sikivat, chanteur de la tradition; mais ce chant s'estompe et laisse place au rock qui à la fin s'efface à son tour pour laisser revenir le tambour et le chant personnel ${ }^{15}$. Hommage rendu à la tradition, cette pièce n'est pas une fusion, elle est presque une opposition qui s'intitule «Moi, Groenlandais». Ole Kristiansen, autre chanteur de cette immense île, a, quant à lui, samplé quelques jeux de tambour d'un groupe de Yuit de Sibérie. Il y ajoute bien l'un ou l'autre ayaya mais sa chanson reste fondamentalement occidentale et l'allusion à la tradition est bien trop timide ${ }^{16}$. Relativement nombreux sont ceux qui se risquent simplement à ce genre d'hommages discrets. Le groupe de rap groenlandais, Nuuk Posse, a également introduit quelques frappes de tambour perdues dans la masse d'une musique qui est avant tout hip-hop ${ }^{17}$. Le texte, par contre, mériterait toute notre attention, comme ceux de Sume, de Kristiansen et de la plupart de ces 
groupes et chanteurs, parce qu'ils abordent des sujets graves, des réflexions importantes sur les changements de vie et d'identité. Où est celui qui osera chanter ce mal de vivre, cette interrogation, parfois ces revendications, avec le seul tambour traditionnel? Il y a quelques dix ans, lorsque j'ai procédé à mes premières analyses des chansons inuit actuelles en comparaison avec la tradition, j'ai cru déceler, dans cette masse importante de chansons profondes, les signes avant coureurs d'un renouveau de la chanson traditionnelle. A entendre ce que chantaient alors des portes-paroles comme Charlie Adams au Québec ou Rasmus Lyberth au Groenland, je pensais que l'omniprésence de la guitare et des clichés d'une chanson folk ou rock n'étaient qu'un passage obligé vers une nouvelle autonomie, vers la création d'un mouvement de chanson inuit qui ferait réellement la part des choses. J'entrevoyais l'émergence d'un style, métissé certes, mais un retour au tambour si souvent chanté, si souvent rêvé, appelé. Ce que j'entendais déjà chez certains chanteurs Indiens comme le Montagnais Cyrille Fontaine, je le pensais possible chez les Inuit. Fontaine s'accompagne sur le tambour sacré des Innu pour chanter une chanson qui raconte, qui dénonce, qui dit l'histoire de son peuple. Les Inuit peuvent en faire autant. Pourquoi une chanson comme celle du groupe Fali, Kasper \& Sakka, intitulée "Qilaat» ${ }^{18}$, c'est à dire "le tambour», ne pourrait-elle être chantée sur accompagnement de ce tambour plutôt qu'avec les instruments de la musique pop occidentale? Cette chanson se termine en disant «Laisse le tambour être ta voix, utilise-le au combat. Il est là, tu dois employer le tambour». Pourquoi ne pas le faire alors? La situation actuelle, près de vingt ans plus tard, me fait conclure que le message n'est pas passé. Avec le recul et l'écoute d'un maximum de productions récentes, je constate que la situation n'évolue guère dans le sens espéré. Bien sûr, on voit surgir des artistes intéressants qui se risquent à quelques essais. Lucie Idlout d'Iqaluit est peut-être la chanteuse actuelle la plus prometteuse. Chanteuse Inuit, elle est aussi chanteuse moderne, elle chante le Nunavut autant que le Canada où elle a vécu, plus au Sud. Elle laisse transpirer les innombrables problèmes des jeunes Inuit, le déracinement (comme Lyberth le fait entre Groenland et Danemark), la drogue, le suicide. Elle insiste sur l'importance de la vie à même la terre du Nunavut et sur le respect qu'il faut garder pour cette terre. Une chanson est empreinte de tension dramatique, elle est chantée sur accompagnement d'une frappe régulière et obsédante sur un tambour et d'une basse électrique ${ }^{19}$. Idlout chante en anglais (la plupart des chanteurs ici cités chantent en inuktitut), elle y dit que c'est par son grand-père qu'elle a gardé une croyance pour le tambour. Il s'agit certainement de ce qu'on peut trouver de plus intéressant comme métissage, même si le tambour joué est peut-être une grosse caisse ou un tambour d'emprunt, peu importe, les ayaya répétés et la frappe régulière conservent un style inuit évident au service d'un chant vivant et engagé. 
Fig. 2: Chant à tambour.

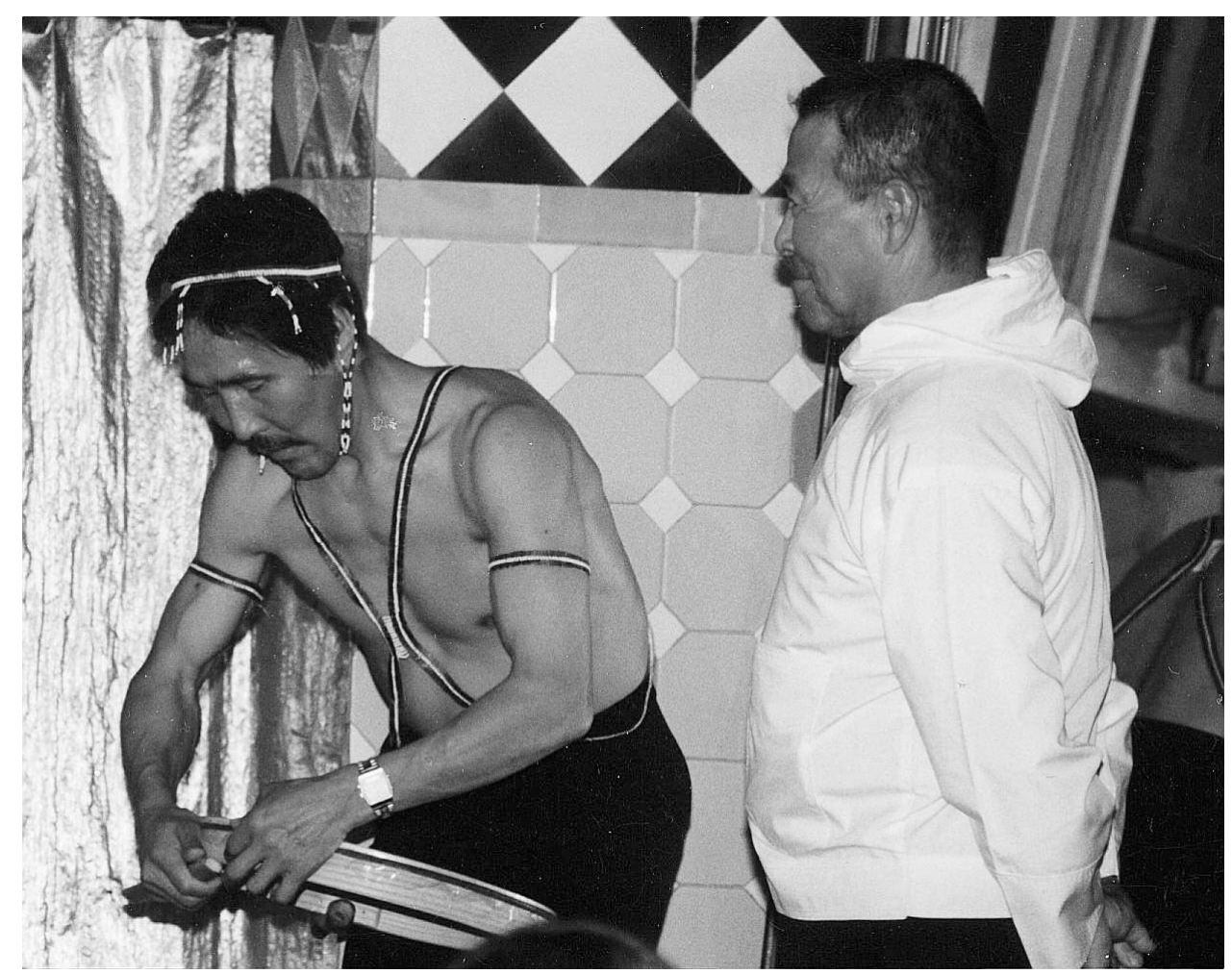

Photo: Jean Delva.

Mais, au-delà des exemples et au-delà de nos aspirations d'Occidentaux à l'écoute et qui plus est assoiffés de comparaisons, il faut sans doute se poser quelques questions fondamentales. Si les métissages sont si rares, si la règle demeure encore et toujours la séparation totale entre expressions traditionnelles et musiques nouvelles, si nous trouvons si peu d'exemples de chants inuits nouveaux qui ne soient pas simplement des copies conformes du rock ou du folk occidental, c'est peut-être dû à quelques raisons profondes. La chanson remplace le chant à tambour, de plus en plus, inexorablement. Après la country, le folk, la pop, s'en viennent le hard-rock et le rap. Les textes jusqu'alors souvent du plus haut intérêt, conservant leur rôle essentiel de véhicules de réflexions et expériences, semblent être en perte de vitesse et basculer eux aussi dans la variété internationale. A titre d'exemple, le dernier grand succès au Groenland est un groupe de hard rock, Siissisoq ${ }^{20}$, qui chante des textes parlant d'animaux sauvages africains!!! Encore faut-il savoir que le leader du groupe a travaillé sur un projet en Afrique et a vu tous ces animaux sur place. Ce n'est pas nécessairement une fiction simpliste mais on s'éloigne certes totalement d'un métissage des expressions inuit. Les autres projets récents affirment parfois réaliser ce métissage. Un disque s'intitule Nanu Disco, In search of the roots. Il prétend nous faire découvrir une fresque musicale sur la vie dans l'Arctique. Un narrateur américain, un producteur danois, des musiciens et chanteurs inuit, un DJ inuit, des samplings de tambours et de chants traditionnels inuit!! «It's just as mixed together as Greenland is» dit le communiqué de presse ${ }^{21}$. Effectivement sans doute mais le résultat est catastrophique. Ca ne ressemble à rien sinon à ces projets fumeux que nous réserve de plus en plus souvent cette mode de soi-disant world music. Et je pourrais vous citer d'autres exemples du même genre, guère plus convaincants. Le constat est donc plus 
négatif aujourd'hui qu'il y a dix ans. Non pas que les traditions disparaissent, elles continuent de vivre et même de voyager. Elles conservent cette discrétion, cette retenue médiatique. Elles se pratiquent toujours au grand jour lors de fêtes, rassemblements, festivals internes aux communautés inuit. Elles vivent côte à côte avec la quantité écrasante de nouvelles chansons et productions qui, elles, ont droit à la médiatisation. Mais le chant à tambour, pour ne citer que lui, n'évolue guère plus ou peu. Il est souvent confiné dans un rôle culturel, souvenir, mémoire des anciens, respect d'un savoir. Les jeunes n'y touchent guère et confient leurs compositions à d'autres styles. Personne n'ose prendre ce tambour ancien, le frapper et chanter ce qu'il ressent aujourd'hui. Les raisons, multiples, en sont peut-être plus évidentes qu'il n'y paraît. Le tambour est sacré. Peut-on vraiment s'en saisir (ou en reconstruire bien sûr) pour chanter autre chose, autrement? Le répertoire des chants personnels ne se composait pas n'importe comment, il demandait un langage précis, que les jeunes ne comprennent plus, une codification, des circonstances de composition et d'exécution qui ont changé. Les jeunes sont distancés, ils ont perdu ce contact complexe avec un répertoire et son instrument. Mais ils ont conservé un respect empreint de spiritualité pour ce tambour qu'ils ont énormément chanté, insistant toujours sur son pouvoir. Un pouvoir lié aux ancêtres, aux anciens, à leur savoir, à leur façon de vivre. La plupart des chansons qui parlent du tambour, parlent des générations précédentes, ceux qui savaient vivre sur la terre, ceux qui ont montré le chemin. De ces chants émanent une nostalgie profonde, un regret, un espoir peut-être, mais souvent une déchirure derrière laquelle se profile l'impossible retour en arrière. Même le métissage, la fusion, n'autorisent guère facilement ce retour, cette reprise en main d'un instrument. Parce qu'il est sacré, parce qu'il est plus facile de jouer de la batterie et de la guitare; comme il est plus facile sans doute de se tourner vers un prêtre ou un médecin que de retourner voir un chaman.

12 Au-delà de ces quelques réflexions, on peut, je pense, aller plus loin encore. La musique, au sens où nous l'entendons, n'occupe certainement pas la même place dans les préoccupations quotidiennes des Inuit. Les expressions musicales ont traditionnellement fait partie d'un ensemble indissociable. Les chants, les danses, les jeux musicaux étaient des éléments «normaux» de la vie de tous les jours et de son organisation saisonnière. Certains chants se pratiquaient tous les jours, instinctivement, comme le langage, faisant partie de l'éducation. D'autres ne se pratiquaient qu'à des occasions rituelles. Ils avaient tous un rôle déterminé, évident, ciment parmi les ciments de la vie d'un groupe, instrument de cohésion et de communication, acte social parmi les actes sociaux. Le rôle toujours dévolu aux expressions traditionnelles a changé en apparence mais pas fondamentalement. Ces expressions continuent de faire partie de la vie des groupes inuit. La chanson moderne, par contre, est arrivée comme un passe-temps supplémentaire, comme une expression de délassement, d'entertainment (ce qui ne signifie pas nécessairement qu'elle n'a aucun rôle culturel). Et les Inuit se composent et se jouent des chansons pour se faire plaisir, pour eux, entre eux. C'est une chanson à consommation interne aussi mais débarrassée d'une bonne part du sens social de la tradition. C'est une chanson que l'on se bricole comme on se sculpte une petite pièce anecdotique pour soi. Alors que pour le marché «blanc», il n'est pas question de sculpter des petites pièces simples et gentilles, il est plus que jamais question de produire de l'art. La chanson moderne n'a pas cette prétention et ne doit pas l'avoir; elle ne répond à aucune demande extérieure, elle n'est pas (ou peu) mise en vente sur un marché mondial. La réelle importance est toujours accordée aux valeurs ancestrales, à la terre, la chasse, la pêche et 
ce que les expressions de la tradition peuvent encore en révéler. Dans les années 1950, de nombreuses familles Inuit furent installées dans le grand Nord canadien pour y servir le Canada soucieux d'y affirmer sa souveraineté. Mary Iqaluk, chanteuse d'Inukjuak, fut déportée par la police montée canadienne vers Resolute Bay. Des années plus tard, elle fut réinstallée à Inukjuak où je l'ai rencontrée avant de travailler avec elle à plusieurs reprises en Europe. Dans un courrier récent, je lui suggérais de me parler de cette déportation, de me raconter ses souvenirs. Elle m'a répondu «je le ferai un jour quand j'aurai le temps («the perfect time»), aujourd'hui j'ai trop de choses à faire». Cette réponse m'a rappelé à l'ordre. Nous n'avons pas les mêmes notions d'urgence. Pour Mary, comme pour la plupart des Inuit, l'urgence est de tous les jours. Il est bien plus important de vivre au maximum le quotidien que de repenser au passé. Il est plus urgent de partir camper sur la toundra dès que les beaux jours arrivent, d'aller à la pêche, de chasser, de vivre et rire avec les voisins, les amis, quitte à s'échanger quelques chants de gorge en passant. Pourquoi s'embarrasser de l'histoire alors qu'on peut se nourrir du présent? La musique s'inscrit sans doute dans cette même logique. Le fils de Mary chante et joue de la guitare; le disque de son groupe est loin d'être bon. Mais c'est une chanson faite au jour le jour, elle n'est pas faite pour rester, elle se mange comme le dernier produit de la pêche. Elle exprime aussi la vie de tous les jours avec la simplicité qui lui colle à la peau. Quant à la chanson traditionnelle, elle vit par la bouche de Mary et de ses amies et elles n'ont aucune inquiétude à ce sujet. Elles la vivent toujours au présent comme tous les autres faits et gestes de leurs vies bien remplies. Cette coexistence entre deux types de musiques est devenue tout aussi normale que celles, quotidiennes aussi, entre tous les éléments de deux mondes différents: nourriture, techniques, habitats, habillements, locomotion,etc. Mais tous ces éléments sont utilisés en un amalgame qui peut paraître anachronique, voire totalement «bordélique», dans un seul et même sens: vivre dans ce grand Nord en se soûlant de cette vie, en profitant de l'instant présent sans renier le passé, en ne s'embarassant pas de faire de l'Art là où il suffit de s'exprimer, ni de réfléchir à la musique alors qu'elle est toujours omniprésente sous une forme ou une autre...

Le son du tambour vibrera peut-être un jour dans les mains d'une jeune génération qui lui confiera ses réflexions nouvellement composées, au risque que cet élan nouveau entraîne une certaine désacralisation du genre. Mais apparemment cette urgence n'a pas encore sonné. Peut-être un jour apparaitra-t-elle, forçant l'un ou l'autre jeune chanteur à briser totalement les tabous, à saisir un tambour et à oublier ces années d'impossible métissage. Peut-être! Imaha, disent les Inuit!

\section{NOTES}

1. «Les derniers rois de Thulé» est le titre du livre écrit par Jean Malaurie en 1951, après son premier long séjour au Groenland. Voir Malaurie 1989 [1954].

2. Le père Rousselière, par exemple, fut de ceux qui, au Canada, alla jusqu'à enregistrer les chants traditionnels des Inuit.

3. ULO CD 143. Notice de Leif Immanuelsen.

4. Voir Jennes 1965. 
5. C'est le cas sur l'enregistrement de Ramon Pelinski à Rankin Inlet dans les années 1970; on y entend un hymne chanté par un prédicateur inuit et sa communauté - le chant se termine en une sorte de transe (Musiques et chants Inuit, Eskimo Point et Rankin Inlet, UMMUS UMM202).

6. Sur le disque 33 tours Tumasi Quissa \& Juupie Arnaituk (CBC Northern Services - Gold Records 11.146).

7. Voir Bours 1991.

8. Sur le disque 33 tours Aasivik 77 (ULO 2).

9. Jeu de gorge présent sur le disque 33 tours Nunavut Nukivut. Our land, our strength (ULO32).

10. Sur le disque Chants et jeux des Inuit (Auvidis Unesco D8032).

11. Sur le disque Tudjaat (Columbia CK80226).

12. Interview de Donald Suluk dans Musicworks, the Canadian New Music Periodical, $\mathrm{n}^{\circ} 23$, Spring 1983.

13. Sur le disque Inuit Iglulik (Museum Collection Berlin CD19).

14. Sur le disque Silamiut: Inuugujoq (ULO CD43).

15. Sur la cassette Sume (ULO 1).

16. Sur le disque Ole Kristiansen: Isimiit Iikkamut (ULO CD52).

17. Sur le CD Nuuk Posse (ULO CD105).

18. Chanson présente sur le disque 33 tours Aasivik 79. Qangattarsa qangattarsartigut (ULO 4/5).

19. Sur le disque demo trois titres Lucie Idlout (Amok Artist Agency, Ontario, Canada).

20. Sur le disque Siissisoq (ULO CD141).

21. Sur le disque Nanu Disco. In search of the roots (Sistus CD-13).

\section{RÉSUMÉS}

Les Inuit connaissent depuis longtemps la coexistence de deux mondes et de deux cultures. Ils ont toujours fait preuve d'une immense capacité d'adaptation, plongeant en chacun de ces mondes pour se fabriquer une vie nouvelle et remplacer leur économie de survie. Choisir sans renoncer semble souvent avoir été leur devise. On pourrait dès lors penser qu'en musique également s'est créé, au fil des ans, un ensemble de métissages nouveaux, rencontres ou fusions entre les expressions de la tradition et les éléments importés tels que instruments, gamme, rythmes, fonctions nouvelles et autres conceptions musicologiques ou culturelles. On constate cependant qu'aucun réel mouvement de métissage ne s'est dégagé. D'un côté, les expressions traditionnelles ont continué d'exister, tant bien que mal, bousculées, «défonctionnalisées», mais parfois redynamisées au contact de l'autre monde. De l'autre côté, ont apparu de nombreuses nouvelles musiques: chorales religieuses, musiques de danse, chanson rock et pop, folk et country, grunge et rap. Mais la séparation est la règle, le métissage est l'exception. Le chant traditionnel ne se pratique pas dans un contexte nouveau et les jeunes ne se risquent guère à jouer le tambour des ancêtres pour accompagner leurs chants pourtant inspirés et allant jusqu'à faire référence aux traditions. 


\section{AUTEUR}

\section{ÉTIENNE BOURS}

Etienne Bours est né en 1951. Licencié en droit, il bifurque immédiatement vers la musique. Il travaille pour la Médiathèque de la Commuanuté française de Belgique où il est actuellement conseiller en musiques traditionnelles, chargé de la prospection et des achats de CD pour les seize médiathèques du réseau. Parallèlement à ces activités, il a développé ses passions et recherches personnelles sur un certain nombre de musiques. Voyages en territoire Inuit et Same, organisation de concerts et tournées, organisation d'un festival des Peuples de l'Arctique, conférences et écrits ont jalonné ce parcours. Etienne Bours est également journaliste, écrivant sur les musiques du monde dans Répertoire, Trad Magazine, Imagine et autres revues ou publications ponctuelles. 